\title{
Docência do ensino superior em Enfermagem: uma revisão de literatura
}

\author{
Nursing higher education teaching: a literature review
}

Enseñanza de educación superior en enfermería: una revisión de la literatura

Marielle Cipriano de Moura ${ }^{*}$, Kátia Lima Braga ${ }^{2}$, Bruna Furtado Sena de Queiroz ${ }^{3}$, Francisca Jéssica de Sousa Oliveira ${ }^{4}$, Paulo Ricardo Dias de Sousa ${ }^{4}$, lara Rege Lima Sousa ${ }^{5}$, Annielson de Souza Costa ${ }^{6}$.

\section{RESUMO}

Objetivo: Avaliar o grau de conhecimento que foi produzido sobre a docência. Metodologia: Depois de feita revisão de literatura que teve início após consultas na base de dados, biblioteca virtual em saúde. Os referidos critérios para inclusão foram artigos disponibilizados na íntegra sobre o tema proposto a serem estudados, artigos publicados em português, inglês e espanhol, entre o intervalo de tempo de janeiro de 2014 a dezembro de 2018. De inclusão e de exclusão, resultaram em 14 artigos Resultados: É muito importante que o enfermeiro tenha uma formação construída a partir de elementos práticos agregando o conhecimento técnico - científico que é exigido para as práticas docentes nas instituições de nível superior. Considerações Finais: As atuais perspectivas de formação colocam os processos reflexivos na centralidade do exercício docente.

Palavras-chaves: Docentes de enfermagem, Prática docente de enfermagem, Ensino.

\begin{abstract}
Objective: To evaluate the degree of knowledge that was produced about teaching. Methodology: After a literature review that began after consulting the database, virtual health library. These inclusion criteria were articles made available in full on the proposed theme to be studied, articles published in Portuguese, English and Spanish, from January 2014 to December 2018. Inclusion and exclusion resulted in 14 articles. Results: It is very important that the nurse has a formation built from practical elements aggregating the technical scientific knowledge that is required for teaching practices in higher level institutions. Final Considerations: The current perspectives of formation place the reflexive processes in the centrality of the teaching exercise.
\end{abstract}

Keywords: Nursing teachers, Nursing teaching practice, Teaching.

\section{RESUMEN}

Objetivo: evaluar el grado de conocimiento producido sobre la enseñanza. Metodología: después de una revisión de la literatura que comenzó después de consultar la base de datos, la biblioteca virtual de salud. Estos criterios de inclusión fueron artículos puestos a disposición íntegramente sobre el tema propuesto para ser estudiado, artículos publicados en portugués, inglés y español, desde enero de 2014 hasta diciembre de 2018. La inclusión y exclusión dieron como resultado 14 artículos. Resultados: es muy importante que la enfermera tenga una formación construida a partir de elementos prácticos que agreguen el conocimiento técnico - científico que se requiere para las prácticas docentes en instituciones de nivel superior. Consideraciones finales: Las perspectivas actuales de formación colocan los procesos reflexivos en la centralidad del ejercicio de enseñanza.

Palabras clave: Profesorado de enfermería, Práctica docente de enfermería, Docencia.

\footnotetext{
1Universidade Estadual do Maranhão - UEMA, Timon- Maranhão, *E-mail: mariellecipriano.c@gmail.com

2Instituto de Ensino Superior Múltiplo- IESM

${ }^{3}$ Faculdade Integral Diferencial - FACID-WYDEN, Teresina- Piauí

${ }^{4}$ Centro Universitário Santo Agostinho (UNIFSA), Teresina- Piauí

${ }^{5}$ Centro Universitário UNINOVAFAPI, Teresina- Piauí.

${ }^{6}$ Faculdade de Medicina FMUSP, Universidade de São Paulo, São Paulo-SP.
} 


\section{INTRODUÇÃO}

Atualmente a sociedade vem botando uma série de desafios para as práticas docentes principalmente na área da enfermagem, levando os profissionais a várias reformulações na docência, são feitas exigências no comprometimento com a qualificação e educação. Aprender a ministrar aulas é um processo continuou passivo de mudanças e qualificações precisando atuar na resolução de conflitos, sendo necessário ajustes a qualquer momento, fazendo autoavaliação no dia a dia para a evolução pessoal e profissional (TREVISO P, COSTA BEP, 2017).

Os docentes de universidades e faculdades independente de serem iniciantes ou experientes precisam entender que as práticas docentes se atualizam constantemente essa reflexão é de suma importância pra a pratica adequada docente já que ensinar requer paciência e compreensão e novas atualizações de conhecimentos teórico científicos relacionados as práticas, esse processo exige tempo na formação docente devendo envolver pesquisa, ensino e extensão tendo como um dos principais objetivos de qualificar 0 desempenho do profissional (CUNHA MI, ZANCHET BBA, RIBEIRO GM, 2013).

As práticas pedagógicas docentes na área de enfermagem é necessário o reconhecimento de ações de interdisciplinaridade no modo que as outras áreas envolvidas do conhecimento possam aprofundar guiando o conhecimento de forma adequada, já que a ação docente exige inúmeras práticas e compreensão do que se é passado na teoria e o que realmente acontece, por isso se faz importante a educação permanente e continuada dos profissionais (LAZZARI DD, MARTINI JG, BUSANA JA, 2015).

A docência no nível superior exige uma formação profissional adequada e de qualidade pois é uma grande responsabilidade dos sistemas de educação e das academias de saúde envolvendo portanto uma série de etapas organizadas entre si para a atuação dos docentes, e ainda um grande impacto de qualidade na formação superior (MERIGHI MAB, JESUS MC P, DOMINGOS SRF, OLIVEIRA DM, ITO TN, 2014).

Dessa forma, questionou-se para produção dessa revisão: quais foram os conhecimentos produzidos sobre docência no ensino superior de Enfermagem? Diante disso, o objetivo desse estudo foi analisar o conhecimento produzido sobre a docência no ensino superior em enfermagem.

\section{MÉTODOS}

Para a realização deste estudo optou-se pela revisão da literatura narrativa. Esta investigação teve início após consultas às bases de dados Biblioteca Virtual de Saúde (BVS), SciELO (Scientific Electronic Library Online) e BDENF (Bases de Dados em Enfermagem).

Para o desenvolvimento do referido artigo utilizou-se os descritores em ciências da saúde (DECS): "docentes em enfermagem" $e$ " prática docente de enfermagem " por meio do conector AND. Para guiar a presente revisão foi formulada a seguinte questão: quais os conhecimentos produzidos referente à docência no ensino superior em enfermagem?

Os critérios para se incluir as publicações nesse estudo foram: artigos eletronicamente disponíveis na íntegra sobre o tema, artigos publicados entre janeiro de 2014 e dezembro de 2018. Os dados foram avaliados e organizados em planilhas na ordem numérica no programa Excel 2017.

\section{RESULTADOS}

Foi identificado 44 artigos disponíveis na íntegra, após realizado leitura com ênfase nos critérios de inclusão e exclusão, restaram 14.

A base de dados BVS o maior número de produções nas bases BDENF e SciELO dá ênfase a escassez de produção cientifica sobre o tema na área de enfermagem. Os referidos resultados podem ser atribuídos ao reduzido número de descritores disponíveis sobre o tema.

Os artigos foram dispostos quanto aos autores, título, tipo de estudo, participantes e principais aspectos ou resultados, conforme está descrito no Quadro 1 abaixo. 
Quadro 1 - Artigos utilizados no trabalho de pesquisa.

\begin{tabular}{|c|c|c|c|c|}
\hline AUTORES & TÍTULO & TIPO DE ESTUDO & PARTICIPANTES & PRINCIPAIS ASPECTOS/RESULTADOS \\
\hline $\begin{array}{l}\text { OYAMA } \\
\text { SMR, et al., } \\
2017 .\end{array}$ & $\begin{array}{l}\text { Comunicação do enfermeiro } \\
\text { docente na assistência a } \\
\text { pessoas cegas e surdas }\end{array}$ & $\begin{array}{l}\text { Quantitativo, } \\
\text { descritivo } \\
\text { transversal }\end{array}$ & $\begin{array}{l}\text { Enfermeiros } \\
\text { docentes }\end{array}$ & $\begin{array}{l}\text { A comunicação continua sendo uma problemática importante no acesso } \\
\text { dos surdos e cegos às informações nos serviços de saúde }\end{array}$ \\
\hline $\begin{array}{l}\text { MERIGHII } \\
\text { MAB, et al., } \\
2014 .\end{array}$ & 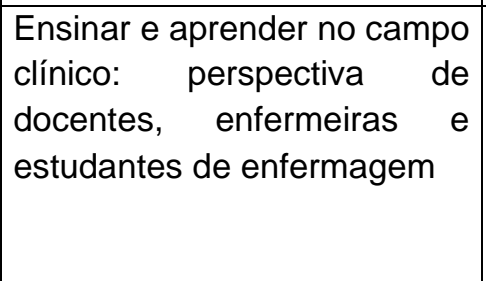 & Qualitativo & $\begin{array}{l}\text { Docentes, } \\
\text { enfermeiros e } \\
\text { estudantes do } \\
\text { curso de } \\
\text { graduação em } \\
\text { enfermagem }\end{array}$ & $\begin{array}{l}\text { Os sujeitos esperam que o ensino clínico subsídio a articulação entre a } \\
\text { teoria e a prática profissional; possibilite o desenvolvimento de uma postura } \\
\text { pró ativa e participativa dos envolvidos na aprendizagem }\end{array}$ \\
\hline $\begin{array}{l}\text { LEITE AS, } \\
\text { TURRINI } \\
\text { RNT; } 2014 .\end{array}$ & $\begin{array}{l}\text { Análise do ensino de } \\
\text { Enfermagem em } \text { Centro } \\
\text { Cirúrgico nas escolas de São } \\
\text { Paulo }\end{array}$ & Quanti-qualitativa & $\begin{array}{l}\text { Coordenadores ou } \\
\text { docentes do curso } \\
\text { de bacharelado } \\
\text { em enfermagem }\end{array}$ & $\begin{array}{l}\text { Há necessidade de aprofundar a discussão sobre o conteúdo mínimo de } \\
\text { CC para a formação do enfermeiro especialista }\end{array}$ \\
\hline $\begin{array}{l}\text { LIMAMM, et } \\
\text { al., } 2016 .\end{array}$ & $\begin{array}{l}\text { Diálogo: rede que entrelaça a } \\
\text { relação pedagógica no ensino } \\
\text { prático- reflexivo }\end{array}$ & Qualitativo & $\begin{array}{l}\text { Docentes de } \\
\text { enfermagem }\end{array}$ & $\begin{array}{l}\text { O diálogo estabelecido na relação pedagógica como } \\
\text { um desafio a ser enfrentado no ensino prático-reflexivo, para que professor } \\
\text { e estudante construam uma relação que estimule o pensar e o agir no } \\
\text { contexto teórico e no campo da prática }\end{array}$ \\
\hline $\begin{array}{l}\text { PERES RR, } \\
\text { et al., } 2015 .\end{array}$ & $\begin{array}{lr}\text { Educação } & \text { ambiental para } \\
\text { docentes } & \text { enfermeiros: } \\
\text { percepção e relação com a } \\
\text { formação do enfermeiro }\end{array}$ & Qualitativo & $\begin{array}{l}\text { Enfermeiros } \\
\text { docentes }\end{array}$ & $\begin{array}{l}\text { A educação ambiental precisa ser fomentada, visando uma formação } \\
\text { comprometida com a sustentabilidade ambiental }\end{array}$ \\
\hline $\begin{array}{l}\text { REIBNITZ } \\
\text { KS, et al., } \\
2016 .\end{array}$ & $\begin{array}{l}\text { Reorientação da formação do } \\
\text { enfermeiro: análise a partir } \\
\text { dos seus protagonistas }\end{array}$ & Estudo de caso & $\begin{array}{l}\text { Profissionais de } \\
\text { saúde, docentes, } \\
\text { alunos e egressos } \\
\text { dos casos }\end{array}$ & $\begin{array}{l}\text { Os reflexos do programa estão presentes na reorientação curricular dos } \\
\text { cursos, estímulo e fortalecimento da educação permanente e integração } \\
\text { ensino-serviço. }\end{array}$ \\
\hline $\begin{array}{l}\text { TONHOM } \\
\text { SFR, et al., } \\
2016 .\end{array}$ & $\begin{array}{|lrr|}\text { Formação } & \text { de } & \text { enfermeiros } \\
\text { centrada } & \text { na } & \text { prática } \\
\text { profissional: } & \text { percepção de } \\
\text { estudantes } & \text { professores }\end{array}$ & Qualitativo & $\begin{array}{l}\text { Estudantes e } \\
\text { professores de } \\
\text { enfermagem }\end{array}$ & $\begin{array}{l}\text { É importante a inserção dos estudantes no contexto hospitalar para o } \\
\text { desenvolvimento da aprendizagem, permitindo maior aproximação com a } \\
\text { realidade da profissão }\end{array}$ \\
\hline
\end{tabular}

REAS/EJCH | Vol.Sup.35 | e936 | DOI: https://doi.org/10.25248/reas.e936.2019 Página 3 de 6 


\section{Revista Eletrônica Acervo Saúde / Electronic Journal Collection Health ｜ ISSN 2178-2091}

\begin{tabular}{|c|c|c|c|c|}
\hline $\begin{array}{l}\text { BACKESVM } \\
\text { S, et al., } \\
2017 .\end{array}$ & $\begin{array}{l}\text { Lee shulman: contribuições } \\
\text { para a investigação da } \\
\text { formação docente } \\
\text { Em } \\
\text { enfermagem e saúde }\end{array}$ & Reflexão & - & $\begin{array}{l}\text { Apresentaram-se os construtos, concluindo que sua utilização na } \\
\text { investigação pode contribuir tanto para a análise da formação quanto da } \\
\text { pratica docente }\end{array}$ \\
\hline $\begin{array}{l}\text { FERNANDE } \\
\text { S MC, et al., } \\
2016 .\end{array}$ & \begin{tabular}{lll|} 
O conteúdo da & saúde & do \\
trabalhador & e & as \\
metodologias de & ensino & na \\
formação do enfermeiro
\end{tabular} & Qualitativo & $\begin{array}{l}\text { Docentes de } \\
\text { enfermagem }\end{array}$ & $\begin{array}{l}\text { Evidenciou-se que o currículo do curso de enfermagem prevê, para a } \\
\text { formação do enfermeiro, os conteúdos essenciais ao campo da Saúde do } \\
\text { Trabalhador. }\end{array}$ \\
\hline $\begin{array}{l}\text { MESQUITA } \\
\text { SKC, et al., } \\
2016 .\end{array}$ & $\begin{array}{l}\text { Metodologias ativas de } \\
\text { ensino/aprendizagem: } \\
\text { dificuldades de docentes de } \\
\text { um curso de enfermagem }\end{array}$ & Qualitativo & $\begin{array}{l}\text { Enfermeiros } \\
\text { docentes }\end{array}$ & $\begin{array}{l}\text { Compreender a utilização de metodologias ativas é fundamental para } \\
\text { atender aos pressupostos do paradigma educacional contemporâneo. }\end{array}$ \\
\hline $\begin{array}{l}\text { SALES } \\
\text { PRSS, et al., } \\
2015 .\end{array}$ & $\begin{array}{l}\text { Integração academia-serviço } \\
\text { na formação de enfermeiros } \\
\text { em um hospital de ensino }\end{array}$ & Qualitativo & $\begin{array}{l}\text { Docentes, } \\
\text { enfermeiros } \\
\text { assistenciais e } \\
\text { estudantes de } \\
\text { enfermagem }\end{array}$ & $\begin{array}{l}\text { Depreende-se a necessidade de investimentos na qualificação do processo } \\
\text { de integração ensino e serviço }\end{array}$ \\
\hline $\begin{array}{l}\text { LEAL LA, et } \\
\text { al., } 2017 .\end{array}$ & $\begin{array}{l}\text { O docente de administração } \\
\text { em enfermagem: formação e } \\
\text { competências profissionais }\end{array}$ & Qualitativo & $\begin{array}{l}\text { Docentes de } \\
\text { enfermagem }\end{array}$ & $\begin{array}{l}\text { Os docentes da área devem repensar sua prática educacional e centros } \\
\text { formadores refletir em sobre o estabelecimento de estratégias pedagógicas } \\
\text { para aprimoramento de competências nos docentes. }\end{array}$ \\
\hline $\begin{array}{l}\text { SILVA EFL, } \\
\text { et al.,2017. }\end{array}$ & $\begin{array}{l}\text { Competências do docente do } \\
\text { ensino clínico no curso de } \\
\text { graduação em enfermagem: } \\
\text { um estudo de caso }\end{array}$ & Qualitativo & $\begin{array}{l}\text { Docentes de } \\
\text { enfermagem }\end{array}$ & $\begin{array}{l}\text { A Escola de Enfermagem Anna Nery estabelece como eixo basilar, em seu } \\
\text { processo formativo, ações capitaneadas entre teoria e prática, geradoras } \\
\text { de movimentos que resultam no desenvolvimento de competências, } \\
\text { requeridas no Curso de Graduação }\end{array}$ \\
\hline
\end{tabular}

Fonte: Dados da pesquisa, 2019. 


\section{DISCUSSÃO}

É muito importante que o enfermeiro tenha uma formação construída a partir de elementos práticos agregando o conhecimento técnico - científico que é exigido para as práticas docentes nas instituições de nível superior, para dessa forma conseguir práticas docentes atuais conseguindo o desenvolvimento maior na vida docente. (PAPASTAVRO E, et al., 2010).

O diálogo estabelecido na relação pedagógica e um desafio a ser enfrentado no ensino prático-reflexivo, para que professor e estudante construam uma relação estimuladora do pensar e do agir, no contexto teórico e no campo da prática. Essa relação deve propiciar a reflexão diante de impasses comportamentais que permeiam o processo de ensino aprendizagem (LIMA MM, et al., 2016).

No contexto da relação pedagógica cabe ao docente encontrar alternativas e sensibilizar-se para a realização do processo reflexivo, fazendo das oportunidades da formação e do serviço um solo fértil para a aquisição de habilidades para a reflexão recíproca na formação do enfermeiro. Trazer para o cotidiano do processo de formação a reflexão sobre os impasses gerados, como por exemplo as ferramentas tecnológicas, propicia que docentes e estudantes encontrem alternativas para que tais impasses se transformem em terreno fértil para o processo de ensino-aprendizagem e em reflexão na ação recíproca (LIMA MM, et al., 2016).

As situações vividas resultam em experiências onde o indivíduo agrega saberes e desenvolve competências estabelecidas no "agir", na sua capacidade de enfrentamento e superação ao longo do tempo. Vale lembrar que, na singularidade de cada indivíduo, manifestam-se atitudes potencialmente mobilizadoras de recursos cognitivos de maior ou menor intensidade (SILVA EFL, et al., 2017).

Dessa maneira, é importante a formação de enfermeiros ativos, críticos, reflexivos, criativos, sobretudo com a certeza de que o aprendizado é um estado dinâmico e sem limites. Nessa conjuntura, o reconhecimento das responsabilidades pelas instituições de ensino superior, juntamente com seus docentes, quanto à formação de enfermeiros competentes que atendam às necessidades requeridas pelo contexto atual aliado à compreensão da importância de pedagogias e metodologias que melhor norteiem a prática docente, contribui para uma compreensão sobre as atividades do professor com qualidades organizativas, críticas e resolutivas (MESQUITA SKC, et al., 2016).

\section{CONSIDERAÇÕES FINAIS}

Ao analisar os artigos sobre a temática da docência em enfermagem no nível superior, pode-se compreender que é necessária a articulação entre a teoria e a prática profissional possibilitando uma relação pedagógica em que o docente encontre alternativas que sensibilize para a realização do processo reflexivo para o desenvolvimento de uma postura pró ativa e participativa dos envolvidos na aprendizagem. As atuais perspectivas de formação colocam os processos reflexivos na centralidade do exercício docente. Como limitação do presente estudo está o baixo número de produções encontradas nos últimos cinco anos que configure um perfil mais atual de publicações sobre a temática. Esta que permeia discussões e 0 desenvolvimento de pesquisas futuras sobre o processo identitário dos docentes de enfermagem.

\section{REFERÊNCIAS}

1. ANDRADE SR, et al. Relacionamento Inter organizacional na integração ensino-serviço de enfermagem na atenção primária à saúde. Rev Bras Enferm, 2014, 67(4): 520-527.

2. CUNHA MI, et al. Qualidade do ensino de graduação: culturas, valores e seleção de professores. Práxis Educativa, 2013 23(2): 115.

3. LAZZARIDD et al. Docência no ensino superior em enfermagem: revisão integrativa de literatura. Ver Gaúcha Enferm, 2015; 36(3):93-101.

4. LIMA MM et al. Dialogue: network that intert wines the pedagogical relationship into the practical-reflective teaching. Rev Bras Enferm, 2016; 69(4): 610-617.

5. MERIGHI MAB et al. Ensinar e aprender no campo clínico: perspectiva de docentes, enfermeiras e estudantes de enfermagem. Rev Bras Enferm., 2014; 67(4): 505-11. 
6. MESQUITA SKC et al. Metodologias ativas de ensino/aprendizagem: dificuldades de docentes de um curso de enfermagem. Trab. Educ. Saúde, Rio de Janeiro, 2016; 14(2): 473-486.

7. PAPASTAVRO E et al. Student nurses experience of learning in the clinical environment. Nurse Educ Pract, 2010; 10(3): 176-182.

8. PIMENTA SG, ANASTASIOU LGC. Docência no ensino superior. 3. ed. São Paulo: Cortez; 2008.

9. REIBNITZ KS et al. Reorientação da formação do enfermeiro: análise a partir dos seus protagonistas. Rev Gaúcha Enferm, 2016; 37.

10. SILVA EFL et al. Competências do docente do ensino clínico no curso de graduação em enfermagem: um estudo de caso. Rev Enferm UFPE, 2017; 11(10): 4118-4125.

11. SOUSA LMM et al. Revisões da literatura científica: tipos, métodos e aplicações em enfermagem. RPER, 2018; 0: 46-54.

12. TREVISO $P$, COSTA BEP. Percepção de profissionais da área da saúde sobre a formação em sua atividade docente. Texto Contexto Enferm, 2017; 26(1).

13. XAVIER IM et al. Diretrizes curriculares nacionais para o curso de graduação em enfermagem. Rev SOBECC., 2001; 6(4): $8-9$.

14. ZABALZA MA. O ensino universitário: seu cenário e seus protagonistas. Porto Alegre: Artmed, 2004. 